\title{
Long noncoding RNA MYOSLID promotes invasion and metastasis by modulating the partial epithelial-mesenchymal transition program in head and neck squamous cell carcinoma
}

Hong-Gang Xiong ${ }^{1,2}$, Hao Li', Yao Xiao', Qi-Chao Yang ${ }^{1}$, Lei-Lei Yang ${ }^{1}$, Lei Chen ${ }^{1}$, Lin-Lin Bu', Wen-Feng Zhang ${ }^{1,2}$, Jia-Li Zhang ${ }^{1,3^{*}}$ and Zhi-Jun Sun ${ }^{1,2^{*}}$

\begin{abstract}
Background: Partial epithelial mesenchymal transition (p-EMT) was found to play a potential role in the initial stage of metastasis in human head and neck squamous cell carcinoma (HNSCC). Some long noncoding RNAs (IncRNAs) have been reported to function as promoters or inhibitors of cancer metastasis. This study aimed to identify p-EMTrelated IncRNAs in HNSCC.

Methods: Differentially expressed IncRNAs (DE-IncRNAs) and mRNAs (DEGs) in HNSCC obtained from The Cancer Genome Atlas (TCGA) were screened out by using the "edgeR" package. DE-IncRNAs in the Oral squamous cell carcinoma (OSCC) IncRNA microarray dataset GSE84805 were screened out by using the "limma" package. Slugrelated IncRNAs were determined by Pearson correlation analysis (|Pearson correlation coefficient $\mid \geq 0.4, p<0.01$ ) based on TCGA. Survival analysis were performed for the overlapping DE-IncRNAs by using the "Survival" package. Gene Ontology (GO) and Kyoto Encyclopedia of Genes and Genomes (KEGG) pathway enrichment analyses were used to predict the potential functions of MYOSLID. RT-qPCR and In Site Hybridization (ISH) were used to explore the MYOSLID expression and its clinical significance in HNSCC specimens. Immunohistochemical staining, siRNA, wound healing assay, transwell assay, and western blot were used to explore the biological function and potential molecular mechanisms.
\end{abstract}

Results: MYOSLID was identified as a Slug-related InCRNA and with prognostic value among the 9 overlapping DEIncRNAs. GO and KEGG analyses revealed that MYOSLID was closely related to important biological processes and pathways that regulate cancer metastasis. The results of univariate and multivariate Cox regression analysis based on TCGA and HNSCC tissue microarray data suggested MYOSLID was an independent prognostic factor. MYOSLID expression in HNSCC was closely correlated with Slug, PDPN and LAMB3. The knockdown of MYOSLID in OSCC cell line significantly inhibited cell migration and invasion compared to those in the control cells. In addition, the knockdown of MYOSLID significantly reduced Slug, PDPN and LAMB3 expression levels. However, the knockdown of MYOSLID had no effect on the expression levels of the EMT biomarkers E-cadherin and Vimentin.

(Continued on next page)

\footnotetext{
* Correspondence: sunzj@whu.edu.cn; jiali_zhang@whu.edu.cn

${ }^{1}$ The State Key Laboratory Breeding Base of Basic Science of Stomatology (Hubei-MOST) \& Key Laboratory of Oral Biomedicine Ministry of Education, School \& Hospital of Stomatology, Wuhan University, Wuhan, China

Full list of author information is available at the end of the article
}

(c) The Author(s). 2019 Open Access This article is distributed under the terms of the Creative Commons Attribution 4.0 International License (http://creativecommons.org/licenses/by/4.0/), which permits unrestricted use, distribution, and reproduction in any medium, provided you give appropriate credit to the original author(s) and the source, provide a link to the Creative Commons license, and indicate if changes were made. The Creative Commons Public Domain Dedication waiver (http://creativecommons.org/publicdomain/zero/1.0/) applies to the data made available in this article, unless otherwise stated. 
(Continued from previous page)

Conclusions: Our study revealed that MYOSLID expression was closely related to the p-EMT program in HNSCC, and it might be a new predictive biomarker for aggressive HNSCC.

Keywords: Long non-coding RNA, Partial epithelial to mesenchymal transition, MYOSLID, Prognostic biomarkers

\section{Background}

Head and neck squamous cell carcinoma (HNSCC) is one of the most common human malignancies worldwide that originate from epithelial tissue, more than $90 \%$ of head and neck cancer are histopathologically squamous cell carcinoma [1]. An important factor that affects the prognosis of HNSCC is metastasis to the regional lymph nodes or distant organs, which has been reported to reduce the 5-year survival rate by $50 \%$ compared with that of patients with early stage disease [2]. Therefore, controlling metastasis is of great importance for fighting cancer. The epithelial to mesenchymal transition (EMT) program has long been considered play an essential role in cancer metastasis [3, 4]. Cancer cells escape from the primary tumor and colonize distant organs to form a second tumor, similar to the process of embryonic cells that travel a long distance and migrate to their destinations and to form an organ [5]. Classical EMT is a complex process in which nonmotile epithelial cells experience a complete loss of apical-basal polarity and cell-cell adhesion properties and transform into mesenchymal cells with the ability to migrate and invade adjacent tissue [3]. The loss of epithelium markers such as E-cadherin and the gain of mesenchymal marker such as Vimentin are the most common hallmarks of EMT [6].

However, scholars have observed that cancer cells at the leading edges of primary tumor in aggressive HNSCC acquire just some of the traits of mesenchymal cells but still retain some of the traits of epithelium cells, which is referred to as partial EMT (p-EMT) $[4,7,8]$. The implications of a p-EMT program in cancer are still poorly understood. Recent studies show that the most possible implications of $\mathrm{p}$ EMT in squamous cell carcinoma included endowing the leading cells of the cancer nests with the ability to guide a collective migration, which was distinct from a single-cell metastasis, that occurs in a coordinated manner while maintaining cell-cell contact [5]. Podoplanins (PDPN) and laminin subunit beta 3 (LAMB3) were considered as p-EMT markers and have been suggested to be related to cancer cell metastasis. Slug was the only activated epithelial to mesenchymal transition-transcription factor (EMT-TF) in p-EMT cells compared with non-p-EMT cells based on a single-cell sequencing technique [8]. Similarly, Slug was also demonstrated to act as a key regulator of the p-EMT program in a 3D Madin-Darby canine kidney (MDCK) tubule-genesis system that modeled the in vivo process of the $\mathrm{p}$-EMT program [9]. Thus, the precise molecular mechanisms that regulate $\mathrm{p}-$ EMT are still not fully understood.
We now understand that lncRNAs driver many important cancer phenotypes by regulating oncogenes or tumor suppressor genes through multiple mechanisms $[10,11]$. Many studies have suggested that lncRNAs have the potential to serve as important biomarkers for cancer diagnosis or therapeutic targets for cancer [12]. At present, there are some lncRNAs that have been found to be related to prognosis and metastasis in HNSCC, such as MIR31HG, UCA1, NEAT1, and EGFR-AS1 [13-16]. As EMT is a key and necessary process in the early stage of cancer metastasis, many lncRNAs have been identified with the ability to affect the EMT program by regulating the expression of Slug in different kinds of cancer [17-19]. Studies have show that Slug not only regulate EMT but also play a role in regulating p-EMT $[8,9]$. However, lncRNAs that regulate the p-EMT program, along with Slug-related lncRNAs, have never been reported.

We aimed to identify lncRNAs involved in p-EMT in HNSCC. First, we screened differential expressed lncRNAs (DE-lncRNAs) and differential expressed mRNAs (DEGs) in HNSCC from The Cancer Genome Atlas (TCGA) and Gene Expression Omnibus (GEO) databases using the bioinformatics methods. Then we identified lncRNAs that were correlated with the p-EMT regulator gene Slug from the DE-lncRNAs identified from TCGA. We discovered that the lncRNA MYOSLID was the only lncRNA with prognostic value that was correlated with Slug. Next, MYOSLID-related mRNAs was analyzed with Gene Ontology (GO) and KEGG (Kyoto Encyclopedia of Genes and Genomes) analysis, and the results showed that MYOSLID is associated with many important biological functions and signaling pathways that related to metastasis. These results suggested that MYOSLID is a molecule with important functions. Finally, MYOSLID expression and its' clinical significance in HNSCC was validated with a human HNSCC microarray, and its potential molecular mechanism was explored in oral squamous cell carcinoma (OSCC) cell lines.

\section{Methods}

\section{TCGA data download and GEO data re-annotation}

The RNA sequence data and relevant clinical information of 502 cases of HNSCC and 44 cases of cancer adjacent normal tissues were download from the TCGA database (https://www.cancer.gov/about-nci/organization/ ccg/research/structural-genomics/tcga/?redirect=true). The primary site of selected HNSCC cases is larynx, floor of 
mouth, tonsil, base of tongue, other and unspecified parts of tongue, nasopharynx, gum, oropharynx, hypohparynx, palate, buccal mucosa, lip and other ill-defined sites in oral cavity and mouth.

An OSCC lncRNA microarray profiling dataset (GSE84805) was obtained from the GEO database (https://www.ncbi.nlm.nih.gov/gds/?term=GSE84805). Six paired OSCC cancer tissues and adjacent normal tissues were analyzed in this array (platform: GLP16956, Agilent045997 Arraystar human IncRNA microarray V3). The GSE84805 lncRNA expression profiling data were acquired by probe reannotation. The steps for reannotation are simply described as follows. First, the probe sequences for the Agilent-045997 Arraystar human lncRNA microarray V3 from the Agilent website (http://www.agilent. $\mathrm{com} /$ ) were remapped to the human genome (GRCH38) using the SeqMap tool. The probes that were uniquely mapped to the human genome without mismatch were retained. Secondly, we matched the chromosomal location of the retained probes to the chromosomal location of the lncRNAs from the GENCODE project (https://www.gencodegenes.org, release 28).

\section{HNSCC tissue microarray information and fresh tissue specimen acquisition}

The human HNSCC tissue microarray T15-411 included 90 primary HNSCCs, 8 recurrent HNSCCs, 20 HNSCCs with a preoperative chemoradiotherapy history, 37 metastatic lymph nodes, 5 normal mucosa (MUC) samples, and 35 dysplasia (DYS) samples. The clinicopathological information of the patients included in the microarray T15-411 was reported by Wu et al. [20].

In addition, 15 paired fresh OSCC tissues and adjacent normal tissues were obtained from patients with OSCC who underwent surgery at the Department of Oral Maxillofacial Head and Neck Oncology of the Hospital of Stomatology of Wuhan University during 2018.7-2018.12. The clinical information of the 15 OSCC patients is given in Additional file 1: Table S1. All the patients were primary OSCC patients. Residual blood on the fresh tissues was washed away with saline, and the tissue were immediately stored in $\mathrm{a}-80^{\circ} \mathrm{C}$ refrigerator for total RNA and protein extraction. All patients in this study provided informed consent before surgery. The Medical Ethics Committee of the School and the Hospital of Stomatology of Wuhan University approved the study.

\section{Identification of DEGs, DE-IncRNAs and the generation of a Venn diagram}

Differentially expressed mRNAs and lncRNAs were identified in HNSCC tissues in comparison with normal tissue with data obtained from the TCGA database using the "edgeR" package in R. Differentially expressed lncRNAs were identified in HNSCC tissues in comparison with normal tissues from data obtained from GEO with the accession number GSE84805 using the "limma" package in $\mathrm{R}$. The R scripts of "edgeR" and "limma" were shown in Additional file 2: edgeR.txt and Additional file 3: limma.txt respectively. |Fold Change| $>2$ and adjusted $p<0.05$ were set as the statistical threshold value for differentially expressed mRNAs and lncRNAs. A volcano plot with clustering for the significantly differentially expressed lncRNAs and mRNAs in HNSCC were generated with the "gplots" package in R. A Venn diagram was constructed by using the online website VENNY 2.1.

\section{Target gene prediction}

The coexpression relationships of the lncRNAs and mRNAs were determined by calculating the Pearson correlation coefficients. If the absolute value of the Pearson correlation coefficients between mRNAs and MYOSLID were greater than 0.4, the mRNAs were considered MYOSLIDrelated mRNAs $(p<0.01)$. Similarly, if the absolute value of the Pearson correlation coefficients between lncRNAs and Slug were greater than 0.4, the lncRNAs were considered as Slug-related lncRNAs $(p<0.01)$.

\section{Survival analysis}

Survival analysis was performed for the overlapping lncRNAs obtained from bioinformatics analysis using the "Survival" package in R. Survival analysis for MYOSLID expression in the HNSCC tissue microarray T15411 was performed by using the Kaplan-Meier method. The cut-off value of the MYOSLID expression levels was determined based on its median value. The Mantel-Cox log-rank test was used to compare the differences between the two survival curves. $p<0.05$ was considered to be significant.

\section{Function enrichment analysis}

mRNAs with an absolute value of the Pearson correlation coefficient with MYOSLID that were greater than or equal to 0.3 were included in further function enrichment analysis. The GO and KEGG pathway enrichment analyses were performed with the " $\mathrm{R} \times 64$ 3.4.1" software using the "clusterProfiler" package. The enriched GO terms and KEGG pathways with a $p<0.01$ were considered MYOSLIDrelated biologic processes or signaling pathways.

\section{RT-qPCR}

Total RNA was extracted from tissue samples and cell lines using an RNA prep Pure Tissue Kit and an RNA prep Pure Cell/Bacteria Kit (Tiangen, Beijing, China) according to the manufacturer's protocols. cDNA was synthesized with a PrimeScript ${ }^{\text {mi }}$ RT reagent Kit with gDNA Eraser (Perfect Real Time) (TaKaRa, Dalian, China). Real-time fluorescent quantitative PCR was performed by using the Bio-Rad CFX96 Real-Time PCR Detection 
System (Bio-Rad, USA). The mixture of PCRs contained TB Green ${ }^{\bullet}$ Premix Ex Taq ${ }^{\text {ma }}$ (Tli RNaseH Plus; $12.5 \mu \mathrm{l}$ ), cDNA $(2 \mu \mathrm{l})$, forward primer $(1 \mu \mathrm{l})$, reverse primer $(1 \mu \mathrm{l})$, and RNase-free $\mathrm{H}_{2} \mathrm{O}(8.5 \mu \mathrm{l})$, with a total volume of $20 \mu \mathrm{l}$. The thermal cycling conditions were as follows: $30 \mathrm{~s}$ at $95^{\circ} \mathrm{C}, 5 \mathrm{~s}$ for 40 cycles at $95^{\circ} \mathrm{C}, 30 \mathrm{~s}$ at $60^{\circ} \mathrm{C}$. Target mRNA and lncRNA expression was normalized against GAPDH. The differences between groups were calculated with the comparative Ct method $\left(2^{-\Delta \mathrm{CT}}\right)$. All experiments were performed in triplicate. Primer sequences are shown in Additional file 1: Table S2.

\section{Cell culture}

The HNSCC cell lines Cal27, SCC4 and SCC9 were purchased from the ATCC (American Type Culture Collection). Tca8113 and the normal human immortalized oral keratinocyte line (HIOEC) from primary normal human oral epithelial cells that were infected with HPV16E6E7 were established at the Ninth People's Hospital, Shanghai Jiao Tong University School of Medicine [21]. Cal27, SCC4 and SCC9 were cultured in DMEM/high glucose with $10 \%$ fetal bovine serum (FBS, Gibco, Grand Island, NY, USA) and 1\% streptomycin-penicillin. Tca8113 was cultured in RPMI 1640 (Gibco, USA) supplemented with $10 \%$ FBS (Invitrogen, USA) and 1\% streptomycinpenicillin. HIOEC cells were cultured in a defined keratinocyte serum-free medium (KSFM; GIBCO BRL, USA)

\section{In situ hybridization}

The expression level of MYOSLID in tissues was measured with digoxigenin-labeled antisense oligonucleotide probes. The slides of the HNSCC tissue microarray were dewaxed and rehydrated. The slides were soaked in citrate solution, heated to boiling for antigen retravel, and incubated with proteinase K (Servicebio, Wuhan, China) at $37^{\circ} \mathrm{C}$ for $15 \mathrm{~min}$. Then, the slides were washed 3 times for $15 \mathrm{~min}$ each with $0.1 \mathrm{M}$ PBS. The slides were washed with prehybridization buffer for $30 \mathrm{~min}$ at room temperature before hybridization with the MYOSLID probe (Servicebio, Wuhan, China, $8 \mathrm{ng} / \mathrm{ul}$ ) overnight. The sections were then washed with a gradient-diluted SSC solution at $37^{\circ} \mathrm{C}$ for $10 \mathrm{~min}$ followed by incubation with HRP-labeled mouse anti-digoxigenin (1:1000) (Jackson, USA) for $40 \mathrm{~min}$ at $37^{\circ} \mathrm{C}$. Finally, the hybridization signals were visualized with diaminobenzidine (DAB) chromogenic substrate (Dako). The reaction was stopped by washing with water for $5 \mathrm{~min}$. The slide was counterstained with hematoxylin, sealed with neutral resins, and photographed. The probe sequences for MYOSLID were as follows: 5'-DIG- CAGCCATGTCCTTGCCTTC TGCACACGGTA-DIG - 3'.

\section{Immunohistochemistry and scoring system}

Immunohistochemical (IHC) staining of the paraffinembedded HNSCC tissue microarrays were conducted using primary antibodies against Slug (Cell Signaling Technology, 1:200), LAMB3 (Abcam, 1:250), and Podoplanin/gp36[EPR22182] (Abcam, 1:250), as previously described [22]. An isotype goat IgG antibody was used as a negative control. All slides were scanned with an Aperio ScanScope CS scanner (Vista, CA, USA). The histoscore quantification of each sample was analyzed by the Aperio Quantification software algorithms as previously described [23]. Histoscores of each slide, including ISH stain and IHC stain, were calculated according to the formula $(3+$ percent cells $) \times 3+(2+$ percent cells $) \times$ $2+(1+$ percent cells $) \times 1)$ total intensity/total cell number and were used to assess the histoscore of the pixel quantifications.

\section{siRNA and transfection}

To avoid off-target effects, three siRNAs targeting MYOSLID (GenePharma, Shanghai, China) were synthesized for transfection and to detect the silencing efficiency. Cells were seeded into a 6 -well plate $\left(2 \times 10^{5}\right.$ cells/well). siRNAs were mixed with GP-siRNA-Mate Plus (GenePharma, Shanghai, China) at a ratio of 1:1 and incubated for $15 \mathrm{~min}$ before being diluted with FBSfree culture medium and then delivered to the HNSCC cell lines. After $48 \mathrm{~h}$ of culture, cells were harvested for RNA or protein extraction.

\section{Matrigel invasion assay}

Cell invasion assays were performed with transwell chambers $(8.0 \mu \mathrm{m}$ pore size; Corning, USA), which were coated with $40 \mu \mathrm{l}$ Matrigel (3x dilution; $40 \mu \mathrm{l} /$ well; BD Bioscience), in 24-well plates. After transfection for $36 \mathrm{~h}$, the cells were collected and washed with PBS. Then, the cells were resuspended in serum-free medium. A $200 \mu \mathrm{l}$ cell suspension containing $2.0 \times 10^{5}$ cells were added to the upper chamber, and $600 \mu \mathrm{l}$ DMEM containing 30\% FBS was added to the lower chamber. After $36 \mathrm{~h}$ of incubation, the cells on the top of the membranes were removed with a cotton swab, and then the cells underneath the membranes were fixed in $4 \%$ paraformaldehyde for $20 \mathrm{~min}$ and stained with crystal violet for $30 \mathrm{~min}$. The stained cells that were attached to the membranes were counted under a microscope, and 5 fields of view were randomly selected.

\section{Wound healing assay}

The effect of MYOSLID knockdown on cell migration was evaluated with a wound healing assay. A total of $5 \times$ $10^{5}$ cells were seeded in 6 -well plates containing complete medium and incubated at $37^{\circ} \mathrm{C}$ in an atmosphere of $5 \%$ $\mathrm{CO} 2$ for $24 \mathrm{~h}$. A total of $300 \mu \mathrm{l}$ of solution containing $5 \mu \mathrm{l}$ 
of MYOSLID siRNA and $5 \mu \mathrm{l}$ of GP-siRNA-Mate Plus was added to each group when the cell density reached 60 $70 \%$. A thin scratch was made with a $200 \mu \mathrm{l}$ pipette tip when the cell density reached $90 \%$, and the complete medium was replaced with a serum-free medium. The width of the scratch was recorded at $0 \mathrm{~h}, 24 \mathrm{~h}$ and $48 \mathrm{~h}$ with an inverted microscope (Nikon, Japan) to measure the percentage of the area covered by the migrated cells.

\section{Western blotting}

Cells were lysed in cell lysis buffer (Beyotime Biotechnology, China) containing $1 \mathrm{mM}$ phenylmethylsulfonyl fluoride (PMSF). Total protein concentrations in the supernatant were measured with a bicinchoninic acid assay (BCA) (Beyotime biotechnology, China), and protein (30 $\mu \mathrm{g} / \mathrm{lane})$ was separated on $10 \%$ polyacrylamide gels (Servicebio, Wuhan, China) with electrophoresis and subsequently transferred onto PVDF membranes. Membranes were blocked with 5\% skim milk in TBST at room temperature for $1 \mathrm{~h}$. The membranes were incubated with primary antibodies against Slug (Cell Signaling Technology, 1:2000), LAMB3 (Abcam, 1:1000), Podoplanin/gp36[EPR22182] (Abcam, 1: 2000), E-cadherin (Cell Signaling Technology, 1:1000), and Vimentin (D21H3) XP ${ }^{\circ}$ (Cell Signaling Technology, 1:1000) overnight at $4{ }^{\circ} \mathrm{C}$. After washing three times for $5 \mathrm{~min}$ in TBST, the membranes were incubated for $1 \mathrm{~h}$ at $37^{\circ} \mathrm{C}$ with an HRP-labeled goat anti-rabbit IgG (Proteintech, Wuhan, China) diluted 1:5000 in TBST. Bands were visualized using a WesternBright Sirius Chemiluminescent Detection Kit (Advansta, California, USA). GAPDH protein levels were used as loading controls. The experiments were repeated three times.

\section{Statistical analysis}

SPSS 19.0 software and GraphPad Prism 8.0 were utilized for statistical analysis. The statistical significance of the differences was determined by a paired $t$ test or a Student's $t$ test between two groups. The statistical significance of the differences among three groups was determined by one-way ANOVA. Quantitative data are expressed as the mean \pm standard deviation (SD). Pearson's chi-square test or Fisher's exact test was used to determine the relationship between MYOSLID expression and the clinicopathological features of the HNSCC patients. Univariate and multivariate analyses were performed to test whether MYOSLID was related to OS. Survival analysis was performed with the Kaplan-Meier and log-rank test. $p<0.05$ was considered statistically significant.

\section{Results}

Analysis of the differential expressed IncRNAs with data integrated from the TCGA and GEO databases

A total of 9128 DEGs or DE-lncRNAs, including 5731 upregulated and 3397 downregulated DEGs or DE-lncRNAs, were screened from the TCGA database (|fold change $\mid>2$ and adjusted $p<0.05$ ) (Fig. 1a). A total of 819 DEGs or DE-IncRNAs, including 334 upregulated and 485 downregulated (|fold change $\mid>2$ and adjusted $p<0.05$ ), were screened from the GEO dataset (Fig. 1b). There were 109 overlapping mRNAs or lncRNAs, including 25 mRNAs and 84 lncRNAs, between the two groups (Fig. 1c).

\section{Identification of slug-related IncRNAs in human HNSCC based on the TCGA data}

lncRNAs with a Pearson correlation coefficient $\geq 0.4$ were considered Slug-related lncRNAs. There were 54 lncRNAs identified as Slug-related lncRNAs. There were 9 overlapping lncRNAs between the Slug-related lncRNAs, DEIncRNAs of the TCGA and DE-lncRNAs of the GEO, which were LINC01614, LINC01929, LINC01615, MYOSLID, LINC02154, ITGB1-DT, LINC01998, CYTOR, and LINC01060 (Fig. 1c). The Pearson correlation coefficient between the MYOSLID and Slug expression levels was $0.685(p=8.05 \mathrm{e}-77)$, which was the maximum correlation coefficient among the 54 Slug-related lncRNAs.

\section{Survival analysis screening and the assessment of the clinical significance of MYOSLID expression in HNSCC based on the TCGA database}

Kaplan-Meier and log-rank tests were performed for the survival analysis of the 9 overlapping lncRNAs. The results showed that only MYOSLID expression was related to survival (log-rank (Mantel-Cox) $=8.809, p=0.003$ ) (Fig. 1d).

Then, we downloaded the clinical information from 502 HNSCC patients from TCGA to further assess the clinical significance of MYOSLID expression. Three HNSCC patients who were lacking most clinical information were excluded from the analysis. The clinical characteristics of 499 HNSCC patients are shown in Additional file 1: Table S3. Then, we analyzed the relationship between MYOSLID and the clinical pathologic parameters using the chi-square test. The results showed that MYOSLID expression was upregulated in HNSCC tissues compared with that in normal tissues $(\log \mathrm{FC}=$ $2.870, p<0.001)$. MYOSLID expression was significantly correlated with age $(p=0.036)$, clinical stage $(p=0.026)$ and $\mathrm{T}$ classification $(p=0.013)$, as shown in Table 1 .

The univariate analysis based on the information of the 499 HNSCC patients obtained from TCGA indicated that MYOSLID expression (HR $=1.370,95 \%$ CI [1.012-1.853], $p=0.041)$, gender $(\mathrm{HR}=1.381,95 \% \mathrm{CI}$ [1.024-1.862], $p=$ $0.034)$, and TNM stage $(\mathrm{HR}=1.454,95 \%$ CI $[1.118$ 1.891 ], $p=0.005$ ) were all significantly related to survival. Multivariate Cox regression analysis revealed MYOSLID expression (HR $=1.429,95 \%$ CI [1.045-1.953], $p=0.025)$, gender $(\mathrm{HR}=1.372,95 \% \mathrm{CI}[1.003-1.876], p=0.048)$, T stage $(\mathrm{HR}=1.859,95 \% \mathrm{CI}[1.224-2.823], p=0.004), \mathrm{N}$ 


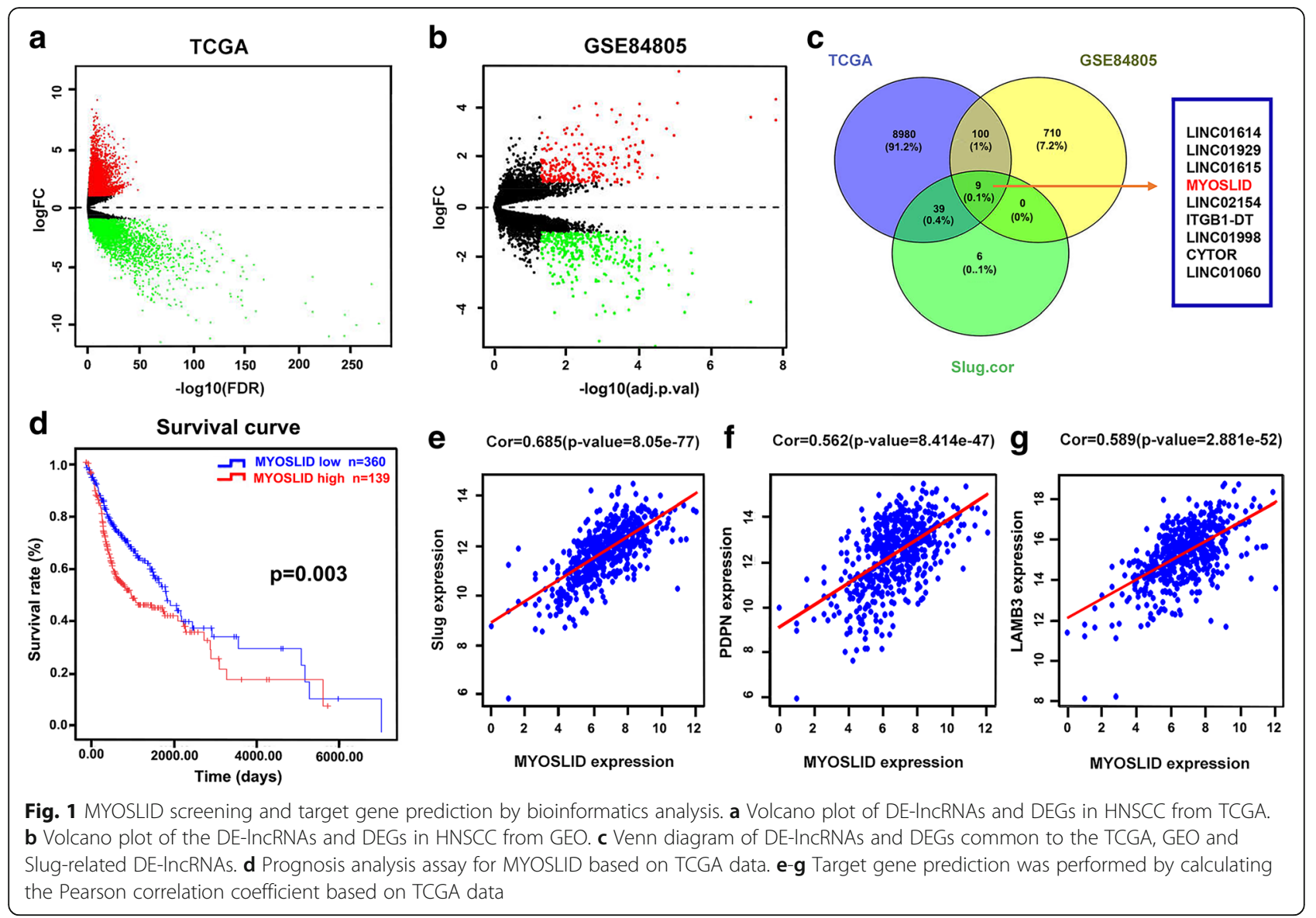

condition $(\mathrm{HR}=1.543,95 \%$ CI $[1.196-1.990], p=0.001)$ and HPV status $(\mathrm{HR}=1.374,95 \%$ CI [1.050-1.798], $p=$ 0.021 ) were independent prognostic factors, as shown in Table 2.

\section{GO and KEGG analysis}

MYOSLID-related mRNAs (|Pearson correlation coefficient $\mid>0.3$ ) were selected for GO and KEGG pathway analysis to further explore their biological function. A total of $24 \mathrm{GO}$ terms and 33 pathways $(p<0.05)$ were identified, as shown in Fig. 2, Additional file 1: Table S4 and S5. The most significantly enriched GO terms for MYOSLID were cell adhesion molecule binding, cadherin binding and protein heterodimerization, as shown in Fig. 2a. Similarly, the significant pathways for MYOSLID-related mRNAs were mainly enriched in the PI3K/AKT signaling pathway, human papillomavirus infection, focal adhesion and regulation of the actin cytoskeleton, as shown in Fig. $2 \mathrm{~b}$.

\section{Expression of MYOSLID in fresh OSCC tissue specimens and OSCC cell lines}

We detected MYOSLID expression in 15 paired fresh OSCC cancer tissues and adjacent normal tissues by RTqPCR. The results showed that MYOSLID expression in the cancer group $(0.01127 \pm 0.01655)$ was higher than that in the adjacent cancer normal tissues $(0.00299 \pm$ $0.00608)(p=0.037)$ (Fig. 3a). Meanwhile, we detected the MYOSLID expression levels in a normal oral epithelium cell line, HIOEC, and 4 OSCC cell lines, Cal27, Tca8113, SCC4, and SCC9. The results showed that the Cal27 cell line had a higher MYOSLID expression level $(0.49254 \pm 0.07004)$ compared with that in Tca8113 $(0.20549 \pm 0.01073)$, SCC4 $(0.02700 \pm 0.00008)$, and SCC9 $(0.03700 \pm 0.00009) \quad(p<0.05)$. Unexpectedly, we found that the human normal oral epithelium cell line HIOEC had the highest MYOSLID expression levels of all of the cell lines that were examined (Fig. 3c).

\section{Evaluation of the clinical significance of MYOSLID} expression in a human HNSCC tissue microarray

The results of the Kaplan-Meier survival analysis showed that patients with higher MYOSLID expression had a poor prognosis (log rank [Mantel-Cox] $=4.858, p=0.028$ ), as show in Fig. 3b.

The results of chi-square $\chi^{2}$ showed that there were no significant relationship between MYOSLID expression and the clinical pathological characteristics (Table 3). However, the results of the ISH histoscore of MYOSLID 
Table 1 Relationship between MYOSLID expression and clinicopathological Characteristics of HNSCC patients based on TCGA $(n=499)$

\begin{tabular}{|c|c|c|c|c|}
\hline \multirow[t]{2}{*}{ Characteristics } & \multicolumn{2}{|l|}{ MYOSLID } & \multirow[t]{2}{*}{$x^{2}$} & \multirow{2}{*}{$\begin{array}{l}p \\
\text { value }\end{array}$} \\
\hline & $\begin{array}{l}\text { Low expression } \\
(n=360)\end{array}$ & $\begin{array}{l}\text { High expression } \\
(n=139)\end{array}$ & & \\
\hline \multicolumn{5}{|l|}{ Age(y) } \\
\hline$<60$ & 149 & 72 & 4.404 & $0.036^{*}$ \\
\hline$\geq 60$ & 211 & 67 & & \\
\hline \multicolumn{5}{|l|}{ Gender } \\
\hline Male & 257 & 109 & 2.534 & 0.111 \\
\hline Female & 103 & 30 & & \\
\hline \multicolumn{5}{|l|}{ Race } \\
\hline White & 312 & 115 & 1.323 & 0.724 \\
\hline Black & 31 & 16 & & \\
\hline Asian & 7 & 3 & & \\
\hline unknow & 10 & 5 & & \\
\hline \multicolumn{5}{|l|}{ Grade } \\
\hline $\mathrm{G} 1 / 2$ & 256 & 104 & 1.196 & 0.754 \\
\hline $\mathrm{G} 3 / 4$ & 89 & 31 & & \\
\hline GX & 13 & 3 & & \\
\hline unknow & 2 & 1 & & \\
\hline \multicolumn{5}{|l|}{ Stage } \\
\hline Stage $|/| \mid$ & 70 & 26 & 7.272 & $0.026^{*}$ \\
\hline Stage III/IV & 232 & 103 & & \\
\hline unknow & 58 & 10 & & \\
\hline \multicolumn{5}{|l|}{ T } \\
\hline $\mathrm{T}_{1}+\mathrm{T}_{2}$ & 166 & 49 & 8.69 & $0.013^{*}$ \\
\hline$T_{3}+T_{4}$ & 175 & 87 & & \\
\hline unknow & 19 & 3 & & \\
\hline \multicolumn{5}{|l|}{ N } \\
\hline Yes & 167 & 69 & 1.726 & 0.422 \\
\hline No & 173 & 66 & & \\
\hline unknow & 20 & 4 & & \\
\hline \multicolumn{5}{|l|}{ M } \\
\hline Mo & 134 & 51 & 1.227 & 0.747 \\
\hline M1 & 1 & 0 & & \\
\hline$M x$ & 41 & 20 & & \\
\hline unknow & 184 & 68 & & \\
\hline \multicolumn{5}{|l|}{ HPV status } \\
\hline Positive & 29 & 1 & 9.634 & $0.002^{*}$ \\
\hline Negative & 49 & 23 & & \\
\hline unknow & 282 & 115 & & \\
\hline
\end{tabular}

Note: HNSCC patients were divided into MYOSLID low and MYOSLID high group according to the cut off value (median $=219.8$ ) $p<0.05$ was considered statistic significant. Differences among variables were evaluated by $\mathrm{x} 2$ or Fisher's exact $\mathrm{X} 2$-test
Table 2 Univariate and multivariate Cox regression analysis for OS in HNSCC patient based on TCGA $(n=499)$

\begin{tabular}{|c|c|c|c|c|c|c|}
\hline \multirow[t]{2}{*}{ Variable } & \multicolumn{3}{|c|}{ Univariate analysis } & \multicolumn{3}{|c|}{ Multivariate analysis } \\
\hline & $p$ & $\mathrm{HR}$ & $95 \% \mathrm{Cl}$ & $p$ & $\mathrm{HR}$ & $95 \% \mathrm{Cl}$ \\
\hline MYOSLID & 0.041 & 1.370 & $1.012-1.853$ & 0.025 & 1.429 & $1.045-1.953$ \\
\hline Age & 0.088 & 1.287 & $0.963-1.720$ & 0.118 & 1.272 & $0.941-1.719$ \\
\hline Gender & 0.034 & 1.381 & $1.024-1.862$ & 0.048 & 1.372 & $1.003-1.876$ \\
\hline Race & 0.240 & 1.218 & $0.876-1.694$ & 0.808 & & \\
\hline Race (1) & & & & 0.846 & 1.155 & $0.271-4.913$ \\
\hline Race (2) & & & & 0.892 & 1.083 & $0.343-3.419$ \\
\hline Race (3) & & & & 0.608 & 1.381 & $0.403-4.734$ \\
\hline Grade & 0.872 & 0.977 & $0.738-1.293$ & 0.679 & 0.941 & $0.705-1.255$ \\
\hline Stage & 0.127 & 1.175 & $0.955-1.445$ & 0.115 & 0.760 & $0.541-1.069$ \\
\hline $\mathrm{T}$ & 0.005 & 1.454 & $1.118-1.891$ & 0.004 & 1.859 & $1.224-2.823$ \\
\hline N & 0.027 & 1.300 & $1.030-1.640$ & 0.001 & 1.543 & $1.196-1.990$ \\
\hline M & 0.039 & 1.121 & $1.006-1.249$ & 0.179 & 1.080 & $0.965-1.209$ \\
\hline HPV & 0.024 & 1.362 & $1.041-1.782$ & 0.021 & 1.374 & $1.050-1.798$ \\
\hline
\end{tabular}

Notes: OS overall survival, $N$ Regional Lymph Nodes, $T$ Primary Tumor, $M$ distant metastasis

Abbreviations: $H R$ hazard ratio, $\mathrm{Cl}$ confidence interval

Race $=$ White was defined as reference, Race (1) = Black, Race (2) = Asian, Race (3) = unknow

expression showed that MYOSLID $(\mathrm{t}=3.271, p=0.0014)$ and PDPN expression $(t=4.866, p<0.0001)$ in the OSCC group was significantly higher than that in the DYS group. Advanced OSCC patients had higher MYOSLID expression levels than those in early stage patients $(F=6.701$, $p=0.0020$ ) (Fig. 4a). Notably, subcellular localization analysis showed that MYOSID was expressed in both the cytoplasm and nucleus (Fig. 4a).

Univariate analysis indicated that MYOSLID expression $(\mathrm{HR}=2.096,95 \% \mathrm{CI}$ [1.061-4.140], $p=0.033)$ and $\mathrm{N}$ condition $(\mathrm{HR}=2.981,95 \% \mathrm{CI}[1.513-5.875], p=0.002)$ were related to OS in OSCC patients. Multivariate Cox analysis indicated that MYOSLID expression ( $\mathrm{HR}=$ 2.223, 95\%CI [1.079-4.580], $p=0.030$ ) and $\mathrm{N}$ condition $(\mathrm{HR}=2.353,95 \% \mathrm{CI}$ [1.136-4.877], $p=0.021)$ were both independent prognostic risk factors related to OS, as show in Table 4.

The expression levels of MYOSLID were correlated with slug, PDPN and LAMB3 in a human HNSCC tissue microarray

Pearson correlation analysis revealed that the expression level of MYOSLID was correlated with Slug $(r=0.2723$, $p=0.0094)$, PDPN $(r=0.3455, p=0.0009)$ and LAMB3 $(r=0.5644, p=0.0001)$ expression, as shown in Fig. 4c.

\section{Knockdown MYOSLID inhibited OSCC cell migration and invasion}

The expression level of MYOSLID was the highest in Cal27 cell line, and Cal27 cell line had a co-expression 


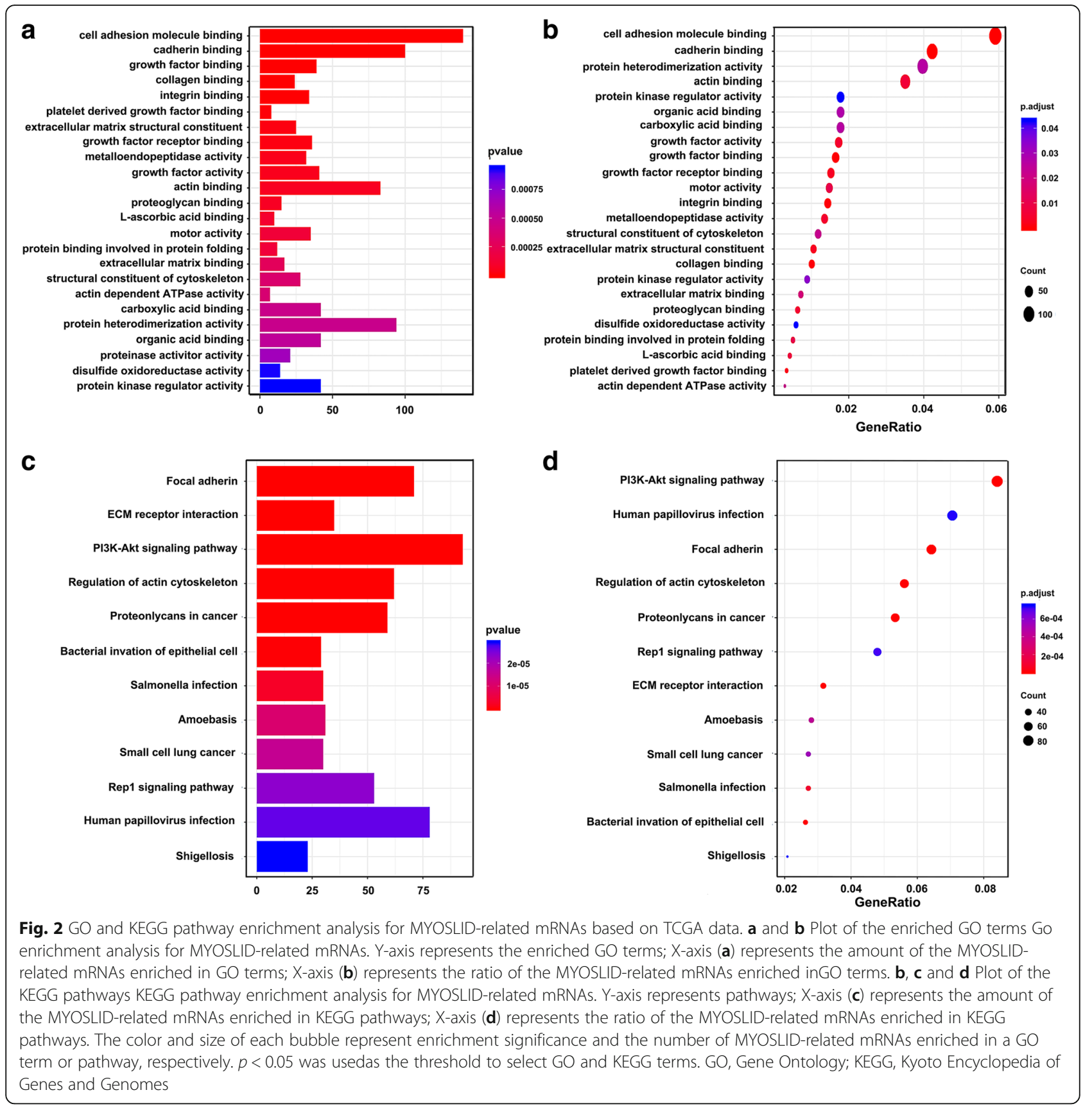

of p-EMT makers (PDPN, LAMB3) and classical EMT markers (E-cadherin, Vimentin) (Fig. 3c, f, g). This indicates that the state of the Cal27 cell line is the closest to a p-EMT. The two siRNA sequences MYOSLID-homo-96 and MYOSLID-homo-232 were selected to knockdown MYOSLID in Cal27 cell lines, as they were validated to have a higher knockout efficiency compared with that of MYOSLID-homo-696 (Fig. 3d). Wound healing assays showed that the knockdown of MYOSLID expression significantly reduced the $48 \mathrm{~h}$ healing rate $(p<0.05$, Fig. $4 \mathrm{a})$. Moreover, the transwell assay showed that the number of cells passed through the filter that was coated with Matrigel of the siRNA groups was much lower than that of the negative control group ( $p<0.05$, Fig. $4 \mathrm{~b})$.

\section{Knockdown MYOSLID inhibited Slug, PDPN and LAMB3 expression}

To further verify the molecular mechanicals of aberrant MYOSLID expression affects the ability of invasion and metastasis of OSCC cells was by regulating the p-EMT program. Expression levels of p-EMT markers Slug, PDPN, LAMB3 and EMT markers E-cadherin, Vimentin 


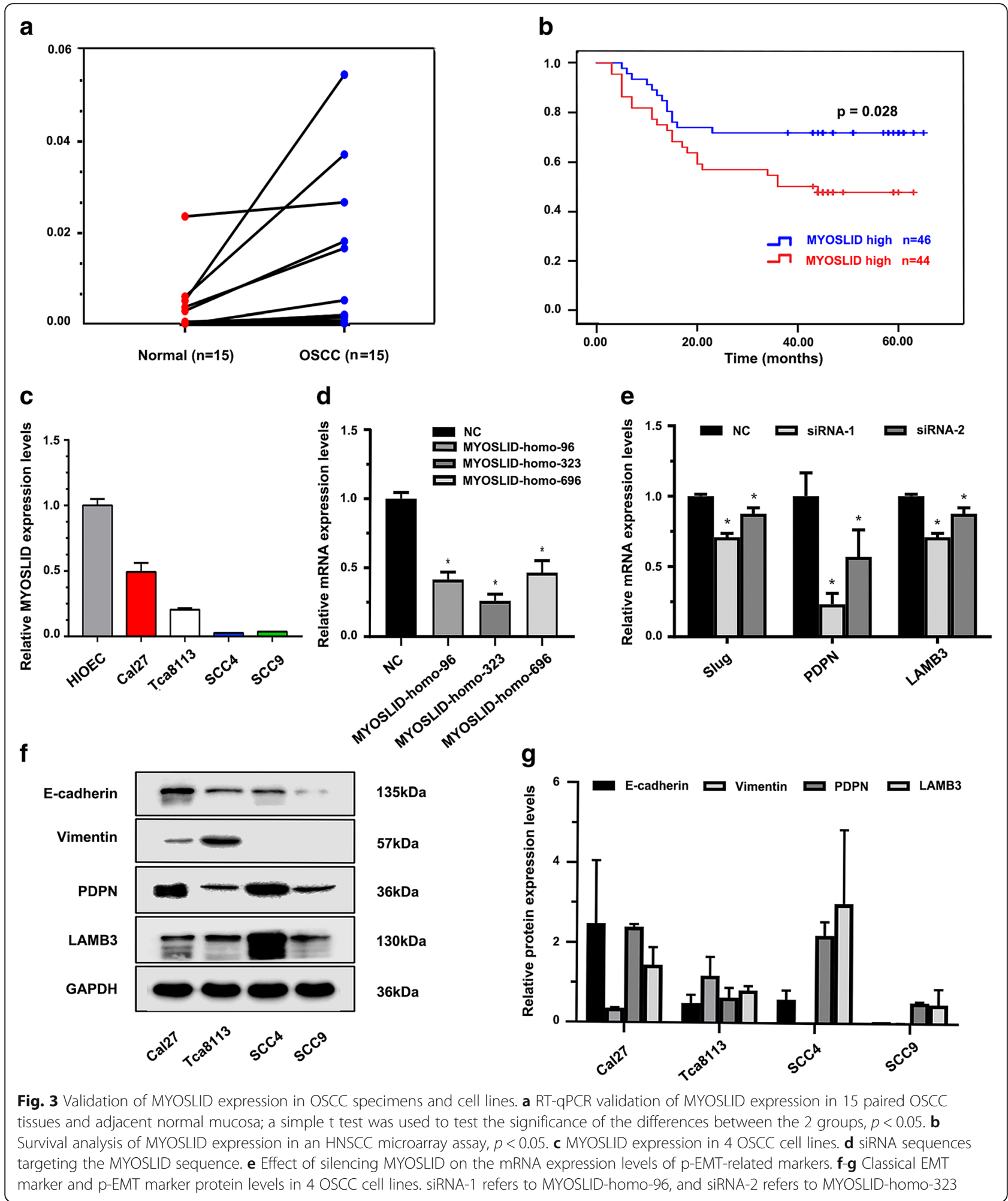

were detected after knockdown MYOSLID in OSCC cell line. The results showed that knockdown MYOSLID expression significantly inhibited Slug, PDPN and LAMB3 mRNA and protein expression levels compared to those in the controls $(p<0.05$, Fig. 3e, Fig. 5c). Interestingly, the expression levels of the classical EMT biomarkers Ecadherin and Vimentin remained unchanged after silencing MYOSLID (Fig. 5c). 
Table 3 MYOSLID expression and clinicopathological Characteristics of patients with $\operatorname{HNSCC}(n=90)$

\begin{tabular}{|c|c|c|c|c|}
\hline \multirow[t]{2}{*}{ Characteristics } & \multicolumn{2}{|l|}{ MYOSLID } & \multirow[t]{2}{*}{$x^{2}$} & \multirow{2}{*}{$\begin{array}{l}p \\
\text { value }\end{array}$} \\
\hline & Low expression $(n=46)$ & High expression $(n=44)$ & & \\
\hline \multicolumn{5}{|l|}{ Age(y) } \\
\hline$<60$ & 23 & 26 & 0.749 & 0.387 \\
\hline$\geq 60$ & 23 & 18 & & \\
\hline \multicolumn{5}{|l|}{ Gender } \\
\hline Male & 41 & 34 & 2.277 & 0.131 \\
\hline Female & 5 & 10 & & \\
\hline \multicolumn{5}{|l|}{ Smoke } \\
\hline Yes & 27 & 20 & 1.580 & 0.209 \\
\hline No & 19 & 24 & & \\
\hline \multicolumn{5}{|l|}{ Drink } \\
\hline Yes & 23 & 21 & 0.046 & 0.829 \\
\hline No & 23 & 23 & & \\
\hline \multicolumn{5}{|l|}{ Stage } \\
\hline Stage $|/| \mid$ & 39 & 38 & 0.045 & 0.831 \\
\hline Stage III/IV & 7 & 6 & & \\
\hline \multicolumn{5}{|l|}{$\mathrm{T}$} \\
\hline $\mathrm{T}_{1}+\mathrm{T}_{2}$ & 27 & 28 & 2.985 & 0.394 \\
\hline$T_{3}+T_{4}$ & 19 & 16 & & \\
\hline \multicolumn{5}{|l|}{ N } \\
\hline Yes & 30 & 25 & 0.472 & 0.492 \\
\hline No & 16 & 18 & & \\
\hline
\end{tabular}

Note: HNSCC patient were divided into MYOSLID low and MYOSLID high group according to the cut off value (median $=2.453$ )

$p<0.05$ was considered statistic significant. Differences among variables were evaluated by $X 2$ or Fisher's exact $X 2$-test

\section{Discussion}

On the one hand, bioinformatics analysis has been widely used to uncover the genetic changes in the highthroughput data from tumors. On the other hand, the pEMT process was observed in a highly invasive HNSCC with a single-cell sequencing technique [8]. The Slug gene was identified as a key gene in the regulation of the p-EMT process [9]. Here, we identified MYOSLID as a lncRNA that was highly correlated with the expression of Slug and with a prognostic value in HNSCC using bioinformatics analysis. GO and KEGG function enrichment analysis of MYOSLID-related mRNAs suggested that MYOSLID expression in HNSCC was associated with many biologic processes, such as cell adhesion molecule binding, cadherin binding, growth factor binding, collagen binding. Most of the enriched pathways or biologic processes have been reported to be involved in regulating cancer metastasis [24-26].

MYOSLID is a long noncoding RNA located in a lncRNA-rich genome region on chromosome 2. Studies on MYOSLID in cancer have never been reported. MYOSLID was first reported as a long noncoding RNA that is related to the differentiation program of vascular smooth muscle cells (VSMCs), and the knockdown of
MYOSLID with siRNA in human coronary artery SMCs (HCASMCs) disrupted the formation of the actin cytoskeleton [27]. Although the function of MYOSLID in epithelial cell types has not been explored, it is reasonable to speculate that MYOSLID expression in epithelial cells may also be related to the cancer cell differentiation program.

This study aimed to understand the function and clinical significance of MYOSLID expression in HNSCC. First, we validated the upregulation of MYOSLID in HNSCC tissues compared with adjacent normal tissue by RT-qPCR. Then, MYOSLID expression was detected in a human HNSCC microarray by ISH. The results showed that MYOSLID expression was associated with poor prognosis in HNSCC. Higher MYOSLID expression was related to advanced TNM stage and lymph node metastasis. This was consistent with the results of bioinformatics analysis of data from TCGA. Therefore, MYOSLID may serves as an oncogene in HNSCC. The function of lncRNA can be predicted by its localization in cells. Subcellular localization analysis showed that MYOSLID was expressed at both cytoplasm and nuclei. However, Zhao et al. reported that most MYOSLID expression was localized in the cytoplasm of VSMCs [27]. This result indicates that MYOSLID may have 


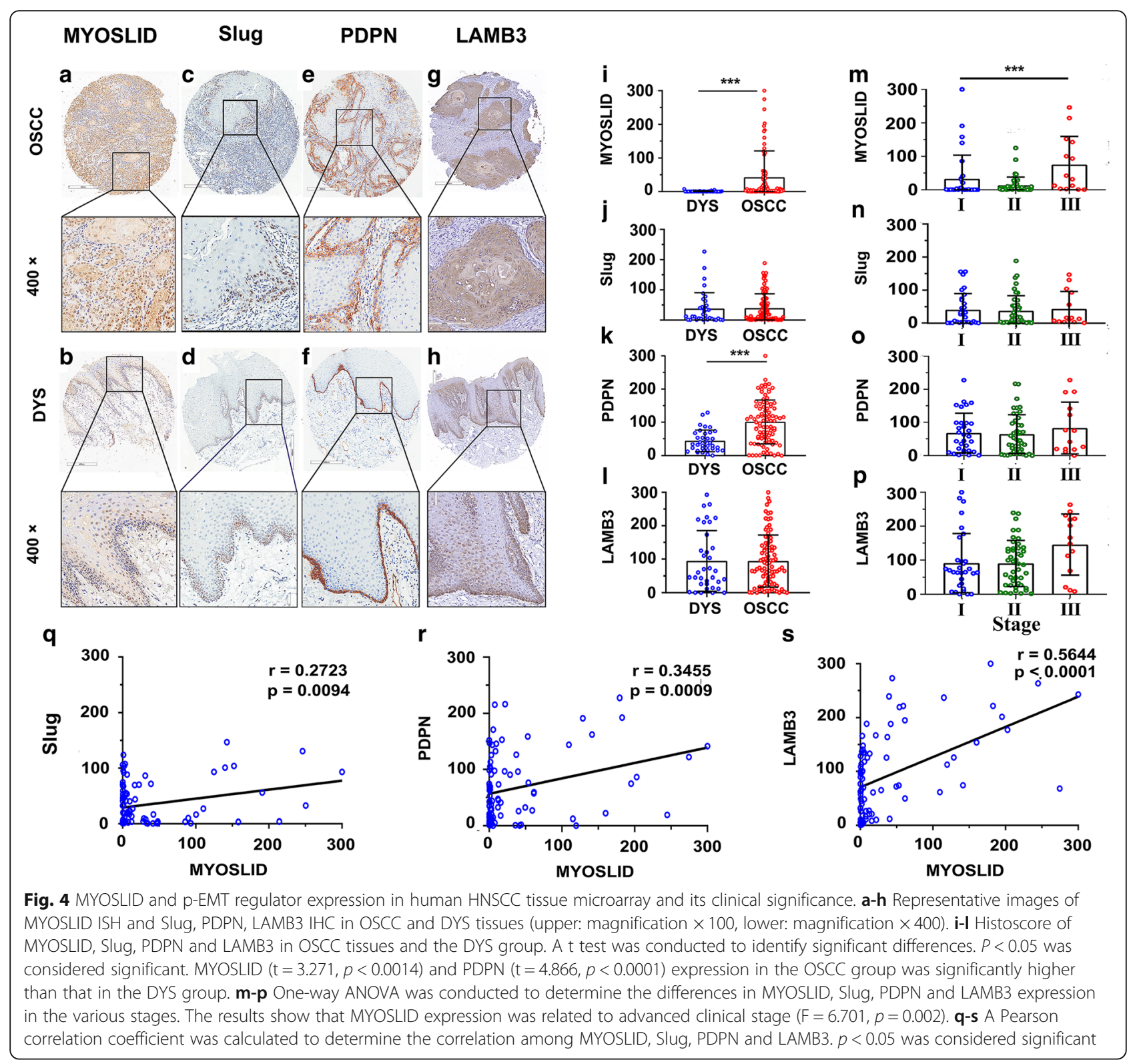

Table 4 Univariate and multivariate Cox regression analysis for OS in HNSCC patients $(n=90)$

\begin{tabular}{|c|c|c|c|c|c|c|}
\hline \multirow[t]{2}{*}{ Variable } & \multicolumn{3}{|c|}{ Univariate analysis } & \multicolumn{3}{|c|}{ Multivariate analysis } \\
\hline & $\bar{p}$ & $\mathrm{HR}$ & $95 \% \mathrm{Cl}$ & $\bar{p}$ & $\mathrm{HR}$ & $95 \% \mathrm{Cl}$ \\
\hline MYOSLID & 0.033 & 2.096 & $1.061-4.140$ & 0.030 & 2.223 & $1.079-4.580$ \\
\hline Age & 0.128 & 1.667 & $0.863-3.219$ & 0.056 & 2.029 & $0.982-4.195$ \\
\hline Gender & 0.130 & 1.793 & $0.843-3.816$ & 0.799 & 1.146 & $0.403-3.252$ \\
\hline Smoke & 0.985 & 1.006 & $0.523-1.936$ & 0.705 & 1.189 & $0.486-2.905$ \\
\hline Drink & 0.193 & 0.641 & $0.328-1.253$ & 0.228 & 0.608 & $0.271-1.366$ \\
\hline Stage & 0.265 & 1.601 & $0.700-3.659$ & 0.333 & 1.575 & $0.628-3.950$ \\
\hline $\mathrm{T}$ & 0.059 & 1.426 & $0.987-2.060$ & 0.101 & 1.469 & $0.928-2.324$ \\
\hline N & 0.002 & 2.981 & $1.513-5.875$ & 0.021 & 2.353 & $1.136-4.877$ \\
\hline
\end{tabular}

Notes: $N$ Regional Lymph Nodes, $T$ Primary Tumor, OS overall survival different functions in cancer cells compared with those in VSMCs.

A list of predicted MYOSLID target genes showed that the Slug gene was at one of the mRNAs that was most strongly related to MYOSLID. Slug is one of the major classical transcription factors (TFs) that drive EMT. Ecadherin, N-cadherin and Vimentin are downstream genes of Slug [28]. However, we did not find E-cadherin, $\mathrm{N}$-cadherin and Vimentin among the list of predicted MYOSLID target genes. Surprisingly, we found that MYOSLID expression was closely correlated with PDPN and LAMB3. Then, we performed IHC for Slug, PDPN and LAMB3 in a human HNSCC microarray. We observed that the PDPN and LAMB3 proteins were 

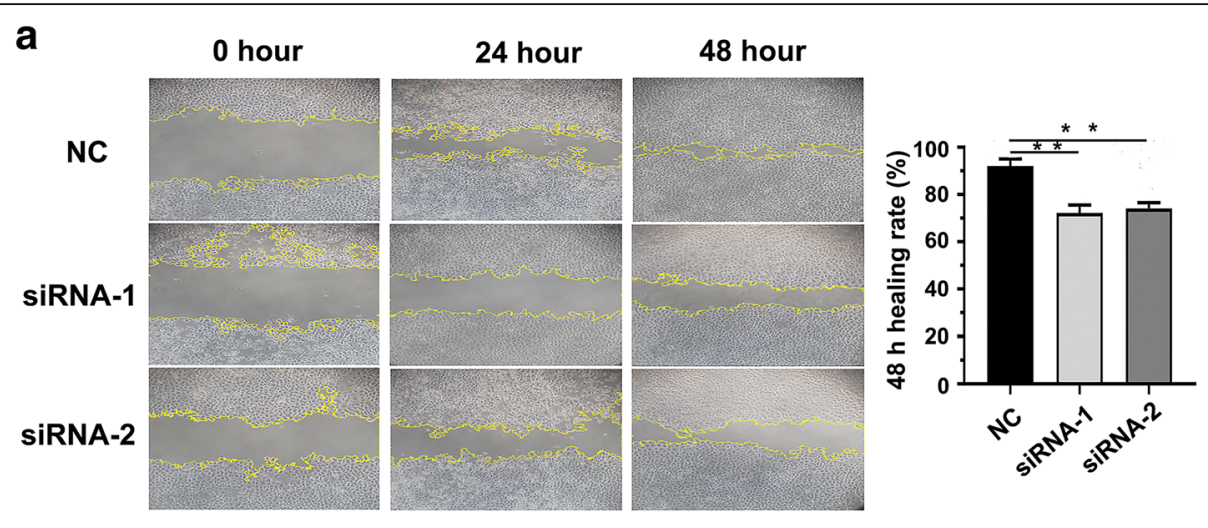

b

Cal27
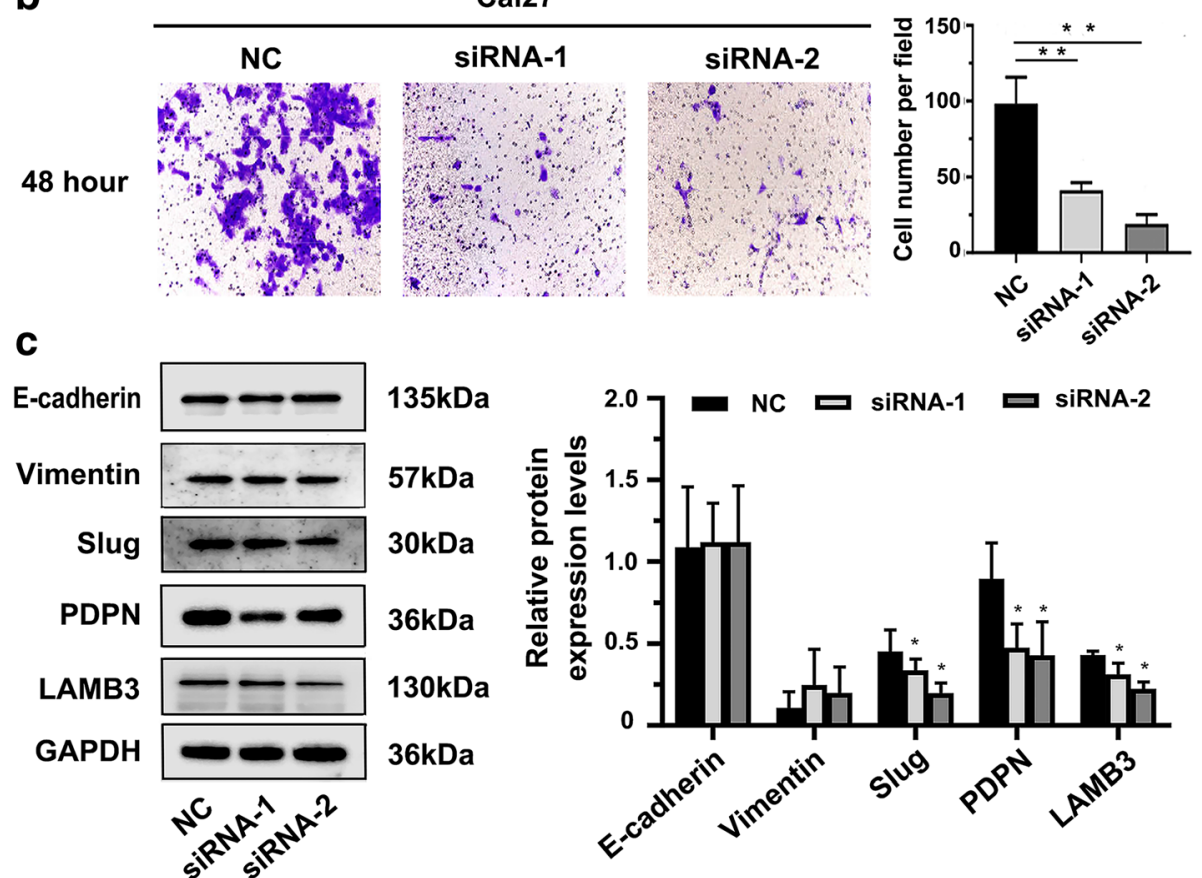

Fig. 5 Effect of MYOSLID on the invasion and metastasis of OSCC cell lines. a Effect of MYOSLID on the cell migration of Cal27, as determined by wound healing assays. Images of the wound healing assays of the 2 siRNA groups and the negative control group were captured at $0 \mathrm{~h}, 24 \mathrm{~h}$ and $48 \mathrm{~h}$ (magnification, $\times 10$ ). Bar graph indicates the mean healing rate of the 3 experimental repetitions of each group. $\mathbf{b}$ Effect of MYOSLID on cell invasion was determined by transwell assay. Representative images of the siRNA group and negative control group were captured after $48 \mathrm{~h}$ (magnification, $\times 20$ ). Bar graph indicates the mean number of cells that passed through the filter coated with Matrigel. Three repeated experiments were performed for each group. c Results of Western blot showed that knockdown MYOSLID in Cal27 cell line caused a significant downregulation of p-EMT related markers Slug, PDPN and LAMB3 protein levels, but protein levels of Classical EMT related markers E-cadherin and Vimentin remain unchanged. "*" indicates that there was statistical significance indicated by a two-sample t test. $p<0.05$ was considered statistically significant. The results are presented as the mean \pm SEM $(n=3)$. siRNA-1 refers to MYOSLID-homo-96, and siRNA-2 refers to MYOSLID-homo-323

specifically expressed in OSCC cells at the tumor periphery. The results of the correlation analysis also indicated that MYOSLID expression was closely correlated with Slug, PDPN and LAMB3. PDPN and LAMB3 are specific p-EMT markers [8]. Leroy $\mathrm{P}$ noted that Slug loses the ability to regulate E-cadherin expression during the p-EMT phase [9]. All of this suggested to us that MYOSLID expression in HNSCC might be related to the function of Slug in controlling the pEMT program instead of the EMT program.
Thus, we further validated and confirmed the functions of MYOSLID in controlling the p-EMT program. We knocked down MYOSLID with siRNA in the Cal27 cell line, as it expressed both the p-EMT markers PDPN and LAMB3 and the epithelial adhesion junction protein E-cadherin. The knockdown of MYOSLID caused a reduction in the expression of Slug, PDPN and LAMB3, but the expression levels of E-cadherin and Vimentin remain unchanged, compared to those in the controls. 
This further supports our previous hypothesis that MYOSLID expression is mainly responsible for maintaining the functions of Slug in regulating the p-EMT program. Wang et al. demonstrate that the knockdown of Slug in the OSCC cell lines UM1 and SCC9 caused an upregulation of E-cadherin and a downregulation of Vimentin. However, Slug knockdown in the SCC15 cell line did not affect E-cadherin and Vimentin expression [29]. This may be explained by the fact that these cell lines are in different EMT phases and that Slug performs different functions. Similarly, our speculation was also supported by Wicki A et al.'s work, in which they overexpressed PDPN in a double transgenic Rip1Podo:Rip1Tag2 mouse model of carcinogenesis to transform a benign adenoma into an invasive carcinoma, in which the complete loss of E-cadherin and the upregulation of $\mathrm{N}$-cadherin were not detected during progression [7]. At present, there are still many unsolved problems regarding the detailed molecular mechanisms of p-EMT. Contrary to our results, some researchers observed different phenomena after deleting PDPN or LAMB3. For example, Asai et al. reported that the downregulation of PDPN in normal human epidermal keratinocytes (NHEKs) caused the upregulation of E-cadherin [30]. Liu et al. reported that the downregulation of LAMB3 in the HNSCC cell lines SNU1041 and SNU1076 increased E-cadherin expression but reduced Vimentin and Slug expression [31]. This may be explained by p-EMT being a metastable and reversable state between the epithelial and mesenchymal states. Inhibiting key genes that control p-EMT will transition the cells back into a non-p-EMT state that is close to the epithelial state.

There are some limitations to this study. We only performed a preliminary study on the functions of MYOSLID in an OSCC cell line that had relatively aggressive features. The specific cells in the p-EMT phase should be sorted for unbiased analysis. The direct interaction between MYOSLID and Slug also needs to be further investigated.

\section{Conclusions}

In summary, we revealed that the upregulation of MYOSLID in HNSCC was associated with poor prognosis. Our results also demonstrated that inhibiting MYOSLID expression significantly inhibited cancer cell invasion and metastasis. Most importantly, the aberrant expression of MYOSLID has no influence on classical EMT markers but does influence the expression levels of pEMT-related markers. This indicates that MYOSLID is a valuable biomarker of aggressive HNSCC. The detection of MYOSLID expression may be meaningful for guiding the selection of clinical treatment options for HNSCC. MYOSLID may be a promising target for controlling cancer metastasis.

\section{Additional files}

Additional file 1: Table S1. Details of the samples used in the RT-qPCR experiment. Table S2. List of primers used for RT-qPCR and sequences of designed MYOSLID small interfere RNA. Table S3. Clinical pathological characteristics of HNSCC patients from the TCGA database $(n=499)$.

Table S4. The GO analysis of the predicted target genes of MYOSLID. Table S5. KEGG pathway analysis of the predicted target genes of MYOSLID. (DOCX 28 kb)

Additional file 2: $\mathrm{R}$ script that use the "edgeR" package for differential expression analysis. (TXT $2 \mathrm{~kb}$ )

Additional file 3: R script that use the "limma" package for differential expression analysis. (TXT $1 \mathrm{~kb}$ )

\section{Abbreviations}

95\%Cl: 95\% confidence interval; DEGs: Differentially expressed mRNAs; DEIncRNAs: Differentially expressed IncRNAs; EMT: Epithelial mesenchymal transition; GEO: Gene Expression Omnibus; GO: Gene Ontology; HNSCC: Head and neck squamous cell carcinoma; HR: Hazard ratio; ISH: In Site Hybridization; KEGG: Kyoto Encyclopedia of Genes and Genomes; LAMB3: Laminin subunit beta 3; MYOSLID: MYOcardin-induced Smooth muscle Long noncoding RNA, Inducer of Differentiation; OS: Overall Survival; OSCC: Oral squamous cell carcinoma; PDPN: Podoplanins; p-EMT: Partial epithelial mesenchymal transition; RT-qPCR: Real time quantitative polymerase chain reaction; TCGA: The Cancer Genome Atlas; TFs: Transcription factors; VSMC: Vascular smooth muscle cell

\section{Acknowledgements}

Not applicable.

\section{Authors' contributions}

HGX, ZJS, JLZ, ZWF designed the study. HGX contribute to the bioinformatics analysis. HGX, HL, YX, QCY and JLZ contributed to the experiments, data acquisition and analysis. LLY and LC provided technical and scientific support. HGX drafted the manuscript with the help of all the authors. ZJS revised the manuscript. All authors read and approved the final manuscript.

\section{Funding}

This work was supported by the National Natural Science Foundation of China [grant numbers 81874131, 81672668, 81672667, 81702703] and Hubei Province Nature Science Funds for Distinguished Young Scholar [grant number 2017CFA062]

\section{Availability of data and materials}

All the data and materials supporting the conclusions were included in the main paper.

\section{Ethics approval and consent to participate}

All protocols dealing with the patients conformed to the ethical guidelines of the Helsinki Declaration and were approved by the Medical Ethics Committee of Hospital of Stomatology Wuhan University.

\section{Consent for publication}

Not applicable.

\section{Competing interests}

The authors declare that they have no competing interests.

\section{Author details}

${ }^{1}$ The State Key Laboratory Breeding Base of Basic Science of Stomatology (Hubei-MOST) \& Key Laboratory of Oral Biomedicine Ministry of Education, School \& Hospital of Stomatology, Wuhan University, Wuhan, China.

${ }^{2}$ Department of Oral Maxillofacial-Head Neck Oncology, School and Hospital of Stomatology, Wuhan University, Wuhan, China. ${ }^{3}$ Department of Oral Pathology, School and Hospital of Stomatology, Wuhan University, Wuhan, China. 
Received: 22 April 2019 Accepted: 28 May 2019

Published online: 25 June 2019

\section{References}

1. Chi AC, Day TA, Neville BW. Oral cavity and oropharyngeal squamous cell carcinoma--an update. CA Cancer J Clin. 2015;65(5):401-21.

2. Burusapat $C$, Jarungroongruangchai $W$, Charoenpitakchai M. Prognostic factors of cervical node status in head and neck squamous cell carcinoma. World J Surg Oncol. 2015;13:51

3. Nieto MA, Huang RY, Jackson RA, Thiery JP. EMT:2016. Cell. 2016;166(1):21-45.

4. Lambert AW, Pattabiraman DR, Weinberg RA. Emerging biological principles of metastasis. Cell. 2017;168(4):670-91.

5. Nieto MA. Epithelial plasticity: a common theme in embryonic and cancer cells. Science. 2013;342(6159):1234850.

6. Serrano-Gomez SJ, Maziveyi M, Alahari SK. Regulation of epithelialmesenchymal transition through epigenetic and post-translational modifications. Regulation of epithelial-mesenchymal transition through epigenetic and post-translational modifications. Mol Cancer. 2016;15:18.

7. Wicki A, Lehembre F, Wick N, Hantusch B, Kerjaschki D, Christofori G. Tumor invasion in the absence of epithelial-mesenchymal transition: podoplaninmediated remodeling of the actin cytoskeleton. Cancer Cell. 2006;9(4):261-72.

8. Puram SV, Tirosh I, Parikh AS, et al. Single-cell transcriptomic analysis of primary and metastatic tumor ecosystems in head and neck Cancer. Cell. 2017;171(7):1611-24.

9. Leroy P, Mostov KE. Slug is required for cell survival during partial epithelialmesenchymal transition of HGF-induced tubule-genesis. Mol Biol Cell. 2007; 18(5):1943-52.

10. Schmitt AM, Chang HY. Long noncoding RNAs in Cancer pathways. Cancer Cell. 2016;29(4):452-63.

11. Huarte M. The emerging role of IncRNAs in cancer. Nat Med. 2015;21(11): 1253-61.

12. Ling H, Fabbri M, Calin GA. MicroRNAs and other non-coding RNAs as targets for anticancer drug development. Nat Rev Drug Discov. 2013;12(11): 847-65.

13. Wang R, Ma Z, Feng L, et al. LncRNA MIR31HG targets HIF1A and P21 to facilitate head and neck cancer cell proliferation and tumorigenesis by promoting cell-cycle progression. Mol Cancer. 2018;17(1):162.

14. Qian Y, Liu D, Cao S, et al. Upregulation of the long noncoding RNA UCA1 affects the proliferation, invasion, and survival of hypopharyngeal carcinoma. Mol Cancer. 2017;16(1):68

15. Wang $\mathrm{P}, \mathrm{Wu} \mathrm{T}$, Zhou $\mathrm{H}$, et al. Long noncoding RNA NEAT1 promotes laryngeal squamous cell cancer through regulating miR-107/CDK6 pathway. J Exp Clin Cancer Res. 2016:35:22.

16. Tan DSW, Chong FT, Leong HS, et al. Long noncoding RNA EGFR-AS1 mediates epidermal growth factor receptor addiction and modulates treatment response in squamous cell carcinoma. Nat Med. 2017;23(10): $1167-75$.

17. Ma M, Xu H, Liu G, et al. MITA1, a novel energy stress-inducible IncRNA, promotes hepatocellular carcinoma metastasis. Hepatology. 2019. https:// doi.org/10.1002/hep.30602

18. Peng $L$, Jiang $B$, Yuan $X$, et al. Super-enhancer-associated long noncoding RNA HCCL5 is activated by ZEB1 and promotes the malignancy of hepatocellular carcinoma. Cancer Res. 2019;79(3):572-84.

19. Wang ZY, Hu M, Dai MH, et al. Upregulation of the long non-coding RNA AFAP1-AS1 affects the proliferation, invasion and survival of tongue squamous cell carcinoma via the Wnt/ß-catenin signaling pathway. Mol Cancer. 2018;17(1):3.

20. Wu L, Deng WW, Huang CF, et al. Expression of VISTA correlated with immunosuppression and synergized with CD8 to predict survival in human oral squamous cell carcinoma. Cancer Immunol Immunother. 2017;66(5):627-36.

21. Yu ZW, Zhong LP, Ji T, Zhang P, Chen WT, Zhang CP. MicroRNAs contribute to the chemoresistance of cisplatin in tongue squamous cell carcinoma lines. Oral Oncol. 2010;46(4):317-22.

22. Sun ZJ, Zhang L, Hall B, Bian Y, Gutkind JS, Kulkarni AB. Chemopreventive and chemotherapeutic actions of mTOR inhibitor in genetically defined head and neck squamous cell carcinoma mouse model. Clin Cancer Res. 2012;18(19):5304-13.

23. Yu GT, Bu LL, Zhao YY, et al. CTLA4 blockade reduces immature myeloid cells in head and neck squamous cell carcinoma. Oncoimmunology. 2016; 5(6):e1151594
24. Läubli H, Borsig L. Selectins promote tumor metastasis. Semin Cancer Biol. 2010:20(3):169-77.

25. Radinsky R. Growth factors and their receptors in metastasis. Semin Cancer Biol. 1991;2(3):169-77.

26. Eisinger-Mathason TS, Zhang M, Qiu Q, et al. Hypoxia-dependent modification of collagen networks promotes sarcoma metastasis. Cancer Discov. 2013;3(10):1190-205.

27. Zhao J, Zhang W, Lin M, et al. MYOSLID is a novel serum response factordependent long noncoding RNA that amplifies the vascular smooth muscle differentiation program. Arterioscler Thromb Vasc Biol. 2016;36(10):2088-99.

28. Lamouille $S, X u$ J, Derynck R. Molecular mechanisms of epithelialmesenchymal transition. Nat Rev Mol Cell Biol. 2014;15(3):178-96.

29. Wang C, Liu X, Huang $H$, et al. Deregulation of Snai2 is associated with metastasis and poor prognosis in tongue squamous cell carcinoma. Int J Cancer. 2012:130(10):2249-58.

30. Asai J, Hirakawa S, Sakabe J, et al. Platelets regulate the migration of keratinocytes via podoplanin/CLEC-2 signaling during cutaneous wound healing in mice. Am J Pathol. 2016;186:101-8.

31. Liu L, Jung $S N$, Oh C, et al. LAMB3 is associated with disease progression and cisplatin cytotoxic sensitivity in head and neck squamous cell carcinoma. Eur J Surg Oncol. 2019;45(3):359-65.

\section{Publisher's Note}

Springer Nature remains neutral with regard to jurisdictional claims in published maps and institutional affiliations.
Ready to submit your research? Choose BMC and benefit from:

- fast, convenient online submission

- thorough peer review by experienced researchers in your field

- rapid publication on acceptance

- support for research data, including large and complex data types

- gold Open Access which fosters wider collaboration and increased citations

- maximum visibility for your research: over $100 \mathrm{M}$ website views per year

At $\mathrm{BMC}$, research is always in progress.

Learn more biomedcentral.com/submissions 